\title{
Síntese e Avaliação Farmacológica de Chalconas como Novos Candidatos a Prototipos de Fármacos Antitumorais
}

Amanda F. Cidade, Thiago Freitas, Luciano M. Lião, Cláudia Pessoa, Anderson C. S. Feitosa, Assuero Meira \& Gilberto L. B. Aquino

Uma série de 34 derivados chalconas foi sintetizada e as suas atividades citotóxicas avaliadas como potenciais protótipos antitumorais. Estes compostos foram testados para avaliação da atividade antiproliferativa in vitro frente às linhagens tumorais MDAMB-435 (Melanoma Humano), HCT-8 (Cólon) e SF-295 (Sistema Nervoso), utilizado o método do MTT. O objetivo deste estudo foi avaliar a atividade biológica de chalconas com diferentes padrões de substituição nos anéis A e B dos anéis fenila destes compostos. Em resumo, neste estudo 25 derivados chalconas demostraram potencial citotóxico relevante permitindo classifica-los como potenciais agentes anti-câncer.

Palavras-chave: combrestatastatinas; chalconas; atividade antitumoral.

A series of 34 chalcones derivatives was synthesized and their cytotoxic activities evaluated as potential antitumoral. These compounds were tested to evaluate the in vitro antiproliferative activity against the tumor cell lines MDAMB-435 (human melanoma), HCT-8 (colon) and SF-295 (Nervous System), used the method of MTT. The purpose this study was to evaluate the biological activity of chalcones with different substitution patterns on the rings a and B of the phenyl rings of these compounds. In summary, this study 25 demonstrated potent cytotoxic chalcones derivatives allowing relevant classifies them as potential anti-cancer agents.

Keywords: combretastatin A-4; chalcones; antitumor activity. 


\section{Introdução}

O câncer é uma doença que se caracteriza pela perda da capacidade de controlar o processo de replicação celular. Nesse contexto, a replicação descontrolada leva a alterações na fisiologia do local onde o processo se desenvolve, culminando na falência do orgão ${ }^{1}$. Atualmente o câncer é responsável por um em cada quatro óbitos, sendo considerada a segunda causa de morte de morte no mundo ${ }^{2}$. No Brasil, a previsão do Instituto Nacional do Câncer (INCA) para 2013 aponta a ocorrência de 520 mil novos casos, o que reforça a magnitude do problema no país ${ }^{3}$.

Neste cenário, a busca por medicamentos antitumorais tem aumentado objetivando tratamentos mais efetivos e seletivos. Baseadas nos avanços da biologia do câncer, as pesquisas buscam moléculas que atuem por mecanismos específico de combate a doença, como por exemplo a inibição da polimerização de microtubulinas, sistema que interfere na mitose de células eucarióticas afetando o crescimento de tumores e supressão do câncer. Moléculas que atuam por este mecanismo representam grupo das substâncias mais atrativas e promissoras como anticancerígenas de aplicação clínica atualmemte, sendo o paclitaxel (Taxol®) o principal representante dessa classe ${ }^{4}$.

Impulsionados pelas expectativas mundiais, a investigação sobre as propriedades antitumorais de chalconas 1 (figura 1) tem recebido atenção significativa ao longo dos últimos anos, especialmente devido a sua semelhança estrutural com os compostos combretastatinas $^{5,6}$. Essas substâncias foram isoladas da casca da árvore sul africana Combretum caffrum, sendo que o mais ativo destes compostos é a combretastatina A-4 (CA-4) 2, um potente agente citotóxico que inibe a polimerização dos microtubulinas, resultando na paralização do crescimento de células tumorais na fase G2-M ${ }^{7,8}$. Porém, o desenvolvimento do CA-4 como um fármaco antitumoral tem seu potencial comprometido devido a sua baixa solubilidade em meios biológicos, o que tem alimentado as investigações sobre os análogos estruturais destes compostos ${ }^{9-11}$.

Estudos têm demonstrado que chalconas, análogos diretos das combretastatinas, podem se ligar ás tubulinas evitando sua polimerização em microtúbulos, porém os mecanismos ainda são incertos. Modificações estruturais em chalconas conduziram a melhoria na sua biodisponibilidades e alguns derivados mostraram citotoxicidades seletiva contra células tumorais ${ }^{6,8}$, neste sentido a triagem de derivados chalconas que apresentem efeitos sinérgicos para atividades antitubulinas e anticâncer é essencial.
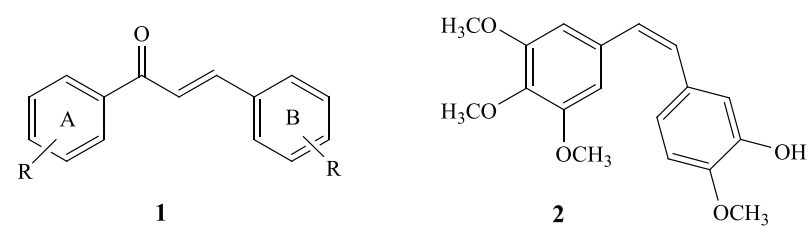

Figura 1. Estrutura geral de chalconas 1, combretastatina A-4 (CA-4) 2.

As chalconas ou 1,3-difenil-2-propen-1-ona 1 são precursores da biosíntese dos flavonóides e isoflavonóides, importantes metabólitos secundários de plantas medicinais. Estes compostos podem ser extraídos de plantas ou obtidos por meios sintéticos, em sua estrutura encontram-se a porção olefínica e a carbonila conjugada ligada a dois anéis fenila ${ }^{12,13}$. Derivados chalconas têm recebido atenção devido as suas estruturas relativamente simples, e em virtude da grande variedade de ações farmacológicas descritas para estes compostos, incluindo atividade bactericida ${ }^{14,15}$, fungicida ${ }^{16}$, antiprotozoária ${ }^{17,18}$, inseticida $^{19}$, antiinflamatória ${ }^{20,21}$, propriedade de inibição enzimática $^{22}$, citotóxica ${ }^{23-26}$ dentre outras atividades ${ }^{12,27}$.

Estudos têm demonstrado que essas atividades biológicas são em grande parte atribuídas a porção olefínica de chalconas, além disso, o padrão de substituição dos anéis A e B de chalconas podem resultar em compostos com propriedades farmacológicas totalmente distintas. A variedade destes compostos é assunto de grande interesse, pois permite o estudo entre estrutura química e atividade biológica conduzindo, portanto, á conclusões úteis á síntese de novos derivados de chalconas antitumorais ${ }^{28}$.

Em virtude da semelhança estrutural das chalconas com os compostos naturais antimitóticos combrestastatinas, realizou-se neste trabalho a síntese bem como a avaliação do potencial citotóxico in vitro de 34 derivados chalconas, frentes as linhagens de células tumorais HCT-8 (cólon), MDA-MB-435 (melanoma) e SF-295 (sistema nervoso). 


\section{Materiais e Métodos}

\section{PROCEDIMENTO GERAL PARA SÍNTESE DE CHALCONAS}

Os derivados chalconas 1-34 foram obtidos via condensação aldólica clássica de Claisen-Schmid, segundo figura 2. Diferentes acetofenonas e benzaldeídos substituíos em quantidades equimolares, foram dissolvidos em solução de $\mathrm{NaOH}(50 \%$ p/v) em metanol. A mistura resultante foi agitada a temperatura ambiente durante 24 horas, após esse tempo vertida em água gelada e neutralizada com solução de $\mathrm{HCl} 50 \%$. O precipitado resultante foi filtrado, lavado com água e recristalizados em etanol ${ }^{12,29}$.

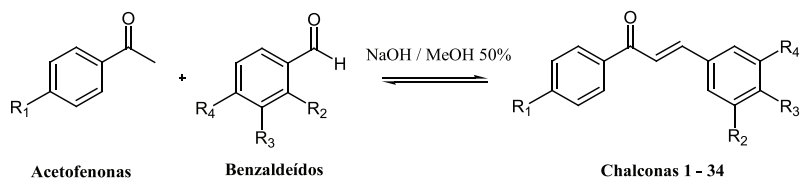

Figura 2. Esquema geral para a síntese dos derivados chalconas 1-34.

\section{LINHAGENS CELULARES, OBTENÇÃO E CULTIVO.}

A avaliação do potencial citotóxico in vitro do composto foi estudado frente às linhagens celulares tumorais humanas MDA-MB-435 (Melanoma), HCT8 (Cólon) e SF-295 (sistema nervoso), realizados no Laboratório de Oncologia Experimental da Universidade Federal do Ceará. Tais linhagens celulares foram cultivadas em meio RPMI 1640, suplementados com $10 \%$ de soro fetal bovino e $1 \%$ de antibióticos, mantidas em estufa a $37^{\circ} \mathrm{C}$ e atmosfera contendo $5 \%$ de $\mathrm{CO}_{2}$.

\section{ENSAIO CITOTÓXICO}

A citotoxicidade foi obtida através do método do $\mathrm{MTT}^{30}$, no qual a quantidade de MTT reduzido a formazan é proporcional ao número de células viáveis. As células tumorais foram distribuídas em multiplacas de 96 poços com densidade de $0,3 \times 10^{6}$ células $/ \mathrm{mL}$, para células suspensas, $0,7 \times 10^{5}$ células $/ \mathrm{mL}$ para as aderidas. Os composto alvo foram testados em concentração única de $5 \mu \mathrm{g}$ e incubado durante 72 horas juntamente com a suspensão de células. Após o período de incubação, $200 \mu \mathrm{L}$ da solução de MTT foram adicionados em cada poço da multiplaca. Depois de um período adicional de 3 horas, $150 \mu 1$ de DMSO foi adicionado em cada poço. Para a quantificação do sal reduzido nas células vivas, as absorbâncias foram lidas com o auxílio do espectrofotometro de placa, no comprimento de onda de $595 \mathrm{~nm}$. A absorbância das multiplacas de controle foi fixada como $100 \%$ e os resultados foram expressos como porcentagem do controle.

\section{Resultado e Discussão}

Com base nos resultados obtidos em estudos anteriores, sintetizamos 34 derivados chalconas com diferentes substituintes no anel A e B, a fim de avaliarmos os efeitos citotóxicos destes derivados. Estas chalconas foram divididas em seis subtipos: metoxiclahconas (110), metilchalconas (11-19), fluorchalconas (20-23), nitrochalconas (24-27), aminochalconas (28 e 29) e benzochalconas de acordo com os substituintes no anel A dos derivados. Todos os compostos sintetizados estão listados na tabela 1 .

Para investigar o efeito antiproliferativo das chalconas (1-34) frente às linhagens tumorais MDAMB-435 (Melanoma - Humano), HCT-8 (Cólon - Humano) e SF-295 (Sistema Nervoso - Humano) foi utilizado o método do $\mathrm{MTT}^{30}$. Este ensaio consiste em uma análise colorimétrica baseada na conversão do sal 3-(4,5-dimetil-2-tiazol)-2,5-difenil-2-H-brometo de tetrazolium (MTT) para formazan, pela atividade da enzima succinil-desidrogenase presente na mitocôndria da célula viável, permitindo dessa maneira quantificar a porcentagem de células vivas.

Para o teste MTT são considerados resultados relevantes os valores de potencial de inibição do crescimento tumoral maiores que $90 \%$ para pelo menos duas das linhagens testadas, neste trabalho foi utilizado como controle positivo a doxorrubicina, fármaco utilizado na terapia contra o câncer. As atividades citotóxicas das amostras frente às três linhagens de células tumorais estão expressas na Tabela 2, com seus respectivos percentuais de inibição (GI\%).

De acordo com os dados da tabela 2, os testes realizados in vitro mostraram notável efeito citotóxico para a maioria dos compostos. Dentre as metoxichalconas (1-10) testadas, os composto $\mathbf{2 , 5}$ e $\mathbf{1 0}$ não demostraram perfis favoráveis de inibição. Por outro lado com o aumento de substituintes 
Tabela 1. Chalconas sintetizadas via condensação de Claisen - Shimidt.

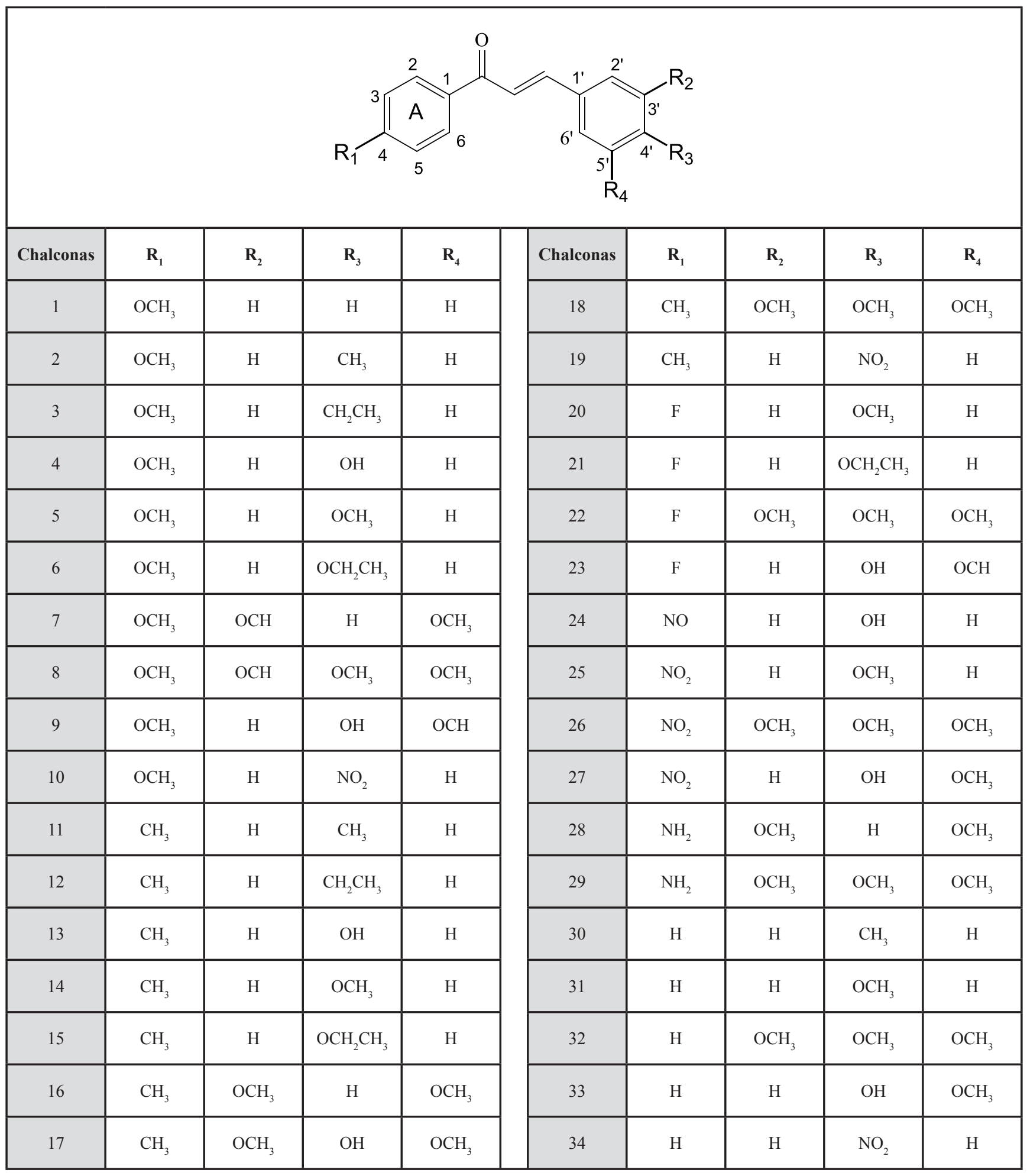


metoxila no anel B $\left(4-\mathrm{OCH}_{3}, 3,5-\mathrm{OCH} 3,3,4,5-\mathrm{OCH}_{3}\right)$ ocorre diminuição da seletividade entre as células e aumento dos percentuais de inibição (GI\%) para as três linhagens. Segundo estudos de relação estrutura atividade entre compostos combrestastatina, unidades metoxila seria um dos requisitos estruturais necessários á atividade antitumoral deste grupo de composto ${ }^{25,28}$, podendo desta forma ser estendido ás atividades das metoxichalconas.

Dentre as metilchalconas (11-19) analisadas, os compostos 11 e 34 não demostraram potencial citotóxico relevante. Porém, uma comparação entre as séries de compostos metoxichalconas (1-10) e metilchalconas (11-19), demostra aumento da atividade antiproliferativa com a inserção de grupos metila na posição 4 do anel A. A introdução de halogênio na posição 4 do anel A das chalconas não altera o percentual de inibição, mantendo o perfil citotóxico considerável das fluorchalconas (20-23), muito embora uma grande número de fármacos utilizados para o tratamento de câncer sejam halogenados ${ }^{32}$.

Para as nitrochalconas apresentadas neste estudo, apenas a chalcona 27 manteve inibição, o que demostra ser desfavorável a inserção deste grupo quando comparado ao controle positivo doxorrubicina.

A introdução do grupo amino no anel A das chalconas, teve um efeito positivo para os composto 28 e 29, o que pode estar aliado á inserção de grupos metoxila nas posições 3', 4' e 5' do anel B, unidade que esta relacionada á impedir a polimerização das microtubulinas $^{6}$. As atividades dos compostos 30 á 34 foram relevantes, o caráter inibitório é mantido apenas com a inserção de substituintes metoxila nas posições 3', 4' e 5' do anel B.

A partir dos resultados expostos acima, 25 análogos chalconas demostraram perfil citotóxico promissor para a atividade antitumoral. As considerações preliminares feitas neste trabalho nos impulsionam a continuar a triagem dentre o compostos apresentados neste estudo, afim de elucidar as características estruturais e físico-químicas necessárias á atividade citotóxica de chalconas frente as linhagens tumorais MDAMB-435, HCT-8 e SF-295.

\section{Conclusão}

$\mathrm{Na}$ presente investigação foram sintetizados 34 derivados chalconas, dos quais 25 destes compostos demostraram relevante potencial citotóxico. Nossos
Tabela 2. Percentuais de inibição (GI\%) das chalconas 1-34, frentes ás células MDA-MB-435, HCT-8 e SF-295 utilizando o método MTT.

\begin{tabular}{|c|c|c|c|}
\hline \multirow{2}{*}{$\mathbf{v}$} & \multicolumn{3}{|c|}{ GI (\%) } \\
\hline & MDA-MB-435 & HCT-8 & SF-295 \\
\hline 1 & 95,84 & 97,8 & 96,27 \\
\hline 2 & 81,53 & 79,49 & 81,16 \\
\hline 3 & 101,84 & 102,59 & 104,25 \\
\hline 4 & 96,64 & 53,75 & 101,39 \\
\hline 5 & 104,4 & 85,3 & 56,8 \\
\hline 6 & 105,13 & 102,48 & 105,64 \\
\hline 7 & 103,68 & 50,82 & 105,9 \\
\hline 8 & 104,56 & 102,31 & 105,99 \\
\hline 9 & 99,12 & 101,13 & 101,47 \\
\hline 10 & 70,62 & 60,88 & 76,28 \\
\hline 11 & 83,74 & 82,48 & 70,59 \\
\hline 12 & 102,24 & 98,48 & 104,6 \\
\hline 13 & 99,2 & 100,23 & 104,08 \\
\hline 14 & 97,84 & 97,01 & 103,9 \\
\hline 15 & 105,77 & 102,87 & 107,37 \\
\hline 16 & 103,6 & 100,79 & 105,12 \\
\hline 17 & 93,43 & 86,31 & 88,12 \\
\hline 18 & 98,16 & 100,62 & 105,9 \\
\hline 19 & 99,76 & 84,9 & 100,17 \\
\hline 20 & 97,76 & 94,82 & 103,73 \\
\hline 21 & 98,4 & 86,48 & 96,88 \\
\hline 22 & 96,8 & 96,39 & 104,34 \\
\hline 23 & 102,8 & 93,86 & 93,41 \\
\hline 24 & 71,39 & 57,13 & 54,2 \\
\hline 25 & 70,84 & 61,96 & 76,13 \\
\hline 26 & 85,35 & 80,28 & 67,73 \\
\hline 27 & 96,48 & 89,69 & 97,48 \\
\hline 28 & 100,46 & 101,16 & 93,09 \\
\hline 29 & 98,64 & 92,62 & 103,38 \\
\hline 30 & 101,84 & 95,38 & 102,34 \\
\hline 31 & 101,44 & 94,42 & 98,53 \\
\hline 32 & 92,31 & 96,62 & 100,17 \\
\hline 33 & 99,44 & 96,68 & 103,12 \\
\hline 34 & 97,6 & 93,86 & 98,87 \\
\hline Doxorrubicina & 97,3 & 96,94 & 87,67 \\
\hline
\end{tabular}


resultados demonstraram que os requisitos estruturais necessárias á atividade antitumoral frente ás células MDAMB-435, HCT-8 e SF-295, podem estar relacionados a inserção de grupos metila e metoxila no anel A das chalconas, e ainda o aumento de grupos metoxila no anel B pode favorecer o perfil citotóxico destes compostos. Este estudo indica que os compostos relevantes deste trabalho podem ser considerados promissores agentes anti-câncer.

\section{Agradecimentos}

Os autores agradecem aos órgãos financiadores FAPEG, CAPES e CNPq.

\section{Referências bibliográficas}

1. Gatenby, R. A.; Gillies, R. J. Nat. Rev. Cancer 2004, 4, 891.

2. http://www.who.int/topics/cancer/en/, acessada em Março 2013.

3. http://www.inca.gov.br/vigilancia/mortalidade, acessada em Março 2013.

4. Altmann, K. H.; Gertsch, J.; Nat. Prod. Rep. 2007, 24, 327.

5. Ducki, S.; Rennison, D.; Woo, M.; Kendall, A.; Chabert, J. F. D.; McGown,A. T.; Lawrence, N.; J. Bioorg. Med.Chem. 2009, 17, 7698.

6. Prakasham, a P.; Saxena, a K.; Luqman, S.; Chanda, D.; Kaur, T.; Gupta, A.; Yadav, D. K.; hanotiya, C. S.; Shanker, K.; Khan, F.; Negi, A. S. J.; Bioorg. Med. Chem 2012, 20, 3049.

7. Kong, Y.; Wang, K.; Edler, M. C.; Hamel, E.; Mooberry, S. L.; Paige, M. a; Brown, M. L.; Bioorg. Med. Chem. 2010, 18, 971.

8. Zhang, H.; Liu, J.-J.; Sun, J.; Yang, X.-H.; Zhao, T.-T.; Lu, X.; Gong, H.-B.; Zhu, H.-L.; Bioorg. Med. Chem. 2012, 20, 3212.

9. Neves, M. P.; Cravo, S.; Lima, R. T.; Vasconcelos, M. H.; Nascimento, M. S. J.; Silva, A. M. S.; Pinto, M.; Cidade, H.; Corrêa, A. G.; Bioorg. Med. Chem. 2012, 20, 25.

10. Boumendjel, A.; Ronot, X.; Boutonnat.; J. Curr. Drug Targ. 2009, 10,363 .

11. Lawrence, N. J.; McGown, A. T.; Curr. Pharm. Des. 2005, 11, 1679.

12. Patil, C. B.; Mahajan, S. K.; Katti, S. A.; J. Pharm. Sci. \& Res. 2009, 1, 11.

13. Katsori, a-M.; Hadjipavlou-Litina, D.; Curr. Med. Chem. 2009, 16,1062 .

14. Latif, F.; Baseer, M.; Iftikhar, F.; Kulsoom, S.; Arkivoc 2009, 20, 318.

15. Sharma, V.; Singh, G.; Kaur, H.; Saxena, A. K.; Ishar, M. P. S.; Bioorg. Med. Chem lett. 2012, 22, 6343.

16. Sivakumar, P. M.; Muthu Kumar, T.; Doble, M.; Chem. Biol. \& Drug Des. 2009, 74, 68.
17. Das, B. C.; Mariappan, G.; Saha, S.; Bhowmik, D.; J. Chem. Pharm. Sci. 2010, 2, 113.

18. Aponte, J. C.; Verástegui, M.; Málaga, E.; Zimic, M.; Quiliano, M.; Vaisberg, A. J.; Gilman, R. H.; Hammond, G. B.; J. Med. Chem. 2008, 51, 6230.

19. Filho, L. S. S.; Plan. Dan. 2007, 25, 747.

20. Yang, H.-M.; Shin, H.-R.; Cho, S.-H.; Bang, S.-C.; Song, G.-Y.; Ju, J.-H.; Kim, M.-K.; Lee, S.-H.; Ryu, J.-C.; Kim, Y.; Jung, S.H.; Bioorg. Med. Chem. 2007, 15, 104.

21. Nowakowska, Z.; Eur. J. Med. Chem. 2007, 42, 125.

22. Najafian, M.; Ebrahim-Habibi, A.; Hezareh, N.; Yaghmaei, P.; Parivar, K.; Larijani, B.; Mol. Biol. Rep. 2011, 38, 1617.

23. Wang, G.; Peng, F.; Cao, D.; Yang, Z.; Han, X.; Liu, J.; Wu, W.; He, L.; Ma, L.; Chen, J.; Sang, Y.; Xiang, M.; Peng, A.; Wei, Y.; Chen, L.; Bioorg. Med. Chem. 2013.

24. Roman, B. I.; Ryck, T. De; Dierickx, L.; Vanhoecke, B. W. a; Katritzky, A. R.; Bracke, M.; Stevens, C. V.; Bioorg. Med. Chem. 2012, 20, 4812 .

25. Hu, Y.; Lu, X.; Chen, K.; Yan, R.; Li, Q.-S.; Zhu, H.-L.; Bioorg. Med. Chem. 2012, 20, 903.

26. John, R.; Sukumarana, K.; Kuttana, G.; Raob, M. N. A.; Subbarajuc, V.; Kuttana, R.; Can. Lett. 1995, 97, 33.

27. Hadjeri,M.; Beney, C.; Boumendjel,A.Cur. Org. Chem. 2003, 7, 679.

28. Bandgar, B. P.; Gawande, S. S.; Bodade, R. G.; Totre, J. V; Khobragade, C. N.; Bioorg. Med. Chem. 2010, 18, 1364.

29. Bukhari, S. N. A.; Jasamai, M.; Jantan, I.; Min. Rev. Med. Chem. 2012, 12, 1394.

30. Mosmann, T.; J. Immunol. Methods, 1983, 65, 55.

31. Chang, J.; Hsieh, H.; Chang, C.; Biol. Pharm. Bull. 2006, 49, 6656.

32. Enmi, K. H.; Iwatashi, Y. H.; Ikita, E. H.; Oyama, N. T.; Irano, T. H.; Biol. Pharm. Bull. 2009, 32, 1109.

\section{Amanda F. Cidade', Thiago Freitas' ${ }^{1}$ Luciano M. Lião², Cláudia Pessoa $^{3}$, Anderson C. S. Feitosa ${ }^{3}$, Assuero Meira ${ }^{3}$ \& Gilberto L. B. Aquino' $^{\prime}$}

${ }^{1}$ Universidade Estadual de Goiás, Unidade Universitária de Ciências Exatas e Tecnológicas, Anápolis, GO

${ }^{2}$ Universidade Federal de Goiás, Campus II Samambaia, CP 131, Goiânia, GO.

${ }^{3}$ Universidade Federal do Ceará, Laboratório de Oncologia Experimental, Fortaleza, CE.

*e-mail: gilberto.benedito@ueg.br 